\title{
ANCIENT CHINESE ASTRONOMICAL OBSERVATIONS RELATED TO THE STELLAR BACKGROUND ON THE SKY
}

\author{
WU SHOUXIAN AND LIU CIYUAN \\ Shaanxi Observatory \\ Academia Sinica \\ Lintong, Shaanxi \\ China, P.R.
}

\section{Stellar reference system in ancient China}

Ancient Chinese astronomers had special interest to measure positions of celestial bodies. "Shishi Xingjing" including at least 115 stars was produced in the 4th century BC. By the 11th century, the measure of stellar coordinates was in full swing and 5 detailed measures were carried on in only 100 years. So several ancient stellar catalogs have been retained up to now. As an ancient reference system, many astronomical phenomena have been recorded on it. This is meaningful for modern astronomers to do some modern research.

An equatorial coordinate system was adopted in ancient China. The whole sky is divided into about 300 asterisms. There is a determinative star in each asterism to determine its position. The twentyeight lunar mansions are 28 asterisms along the equator, which are the basic frame of traditional Chinese coordinate system. Differences of right ascension between the determinative stars of adjoining lunar mansions are called "xiudu" (width of a mansion) which were measured repeatedly in the history. That the winter solstice is in how many degrees of a lunar mansion became the indicator of zero of the system. A statement "an object is in certain degrees of certain mansion (ruxiudu)" means their difference of right ascension. Declination is expressed by the distance from the north pole (qujidu).

Two sets of units were adopted. One set is "degree" $(d u)$. A whole circle is divided into 365.25 "degrees". The other set is a set of length units ( 1 zhang $=10 \mathrm{chi}=100 \mathrm{cun})$.

\section{Positions of moving celestial bodies}

The position measurement for moving bodies is usually related to the stellar background. "Ruxiudu" and "qujidu" were rarely used in these records.

Many eclipses were recorded, but they usually were very simple. "Ruxiudu" were roughly recorded only in a few cases. Lunar and planetary occultations and "fan" (close approaches) of planets or stars occupy an important proportion in ancient astronomical records. Some of them recorded the Moon or a planet covered a certain star or planet; others reported a star or a planet was "fan" by the Moon or another planet, sometimes even with direction and distance measured with "chi, cun". 
Comets are changing their position and shape, therefore their records are detailed. Its date, position, length of tail, and shape are often recorded. Positons usually are expressed by "ruxiudu" or some "chi" to a certain star while length of tail by "zhang, chi, cun". Novas were usually called guest stars. Their positions were reported relative to stars. In meteor records "zhang, chi, cun" often were used to express the positions and lengths.

\section{Research on some terms}

We have seen that the measurement of positions of astronomical phenomena in ancient China was mainly dependent upon the stellar background and expressed by a set of angular units, 1 zhang $=10$ chi $=100$ cun, and some special terms.

Zhang, chi and cun are a set of length units, but they were used as angular units in astronomy for a long time. Their definition has not been found in early books and they are rarely used in the measurement of stars. A book of Qing dynasty (17th century) says one chi is equal to one degree, but modern studies have got different conclusions in which one chi is from 1:24 to 1:5. We consider them unreliable because few and unsuited historical records were used.

It is effective to employ planetary records in this research. We have found 156 planetary records with epochs dated from Han to Yuan dynasty (147 BC to 1364 AD). From these records we get $y=0.14+0.93 x$

that is $1 \mathrm{chi}=0: 93 \pm 0: 04$. The constant 0.14 can be explained by an error of computed position and visual error of observers. Variation dependent on dynasties has not been found. The statistics suggest that as an angular unit one chi is just equal to one degree.

Of terms in ancient records, "fan" was used the most frequently. Meng kang (3rd century) said: fan means within 7 cun, rays touching each other. This definition was mentioned repeatedly in later books. But one chi was repeatedly recorded as the limit of fan in practical historical records. On the other hand, what we need to know is not only the definition of fan, but also the practical situation in ancient observations. For this reason, we have made a statistical analysis of two groups of material: 128 fan of stars by Saturn in "Songshi" (972-1266 AD) and 767 fan of stars by the Moon in "Yuanshi" (1250-1350 AD).

The two bar-graphs (frequency vs distance) gotten from the two groups of records are quite different in their shape, but it can be well explained by the moving velocities of the Moon and Saturn. Both graphs show that most events of fan fall within one degree. From definitions in ancient books and our research on chi, as well as our statistical evaluation of historical records, we can see that the definition of fan which appears frequently in ancient Chinese astronomical records should be one degree. Some error exists in practical observations, but for $98 \%$ of the records it is within 1:25.

Historical astronomical records have offered valuable information for modern astronomy and our work is aimed for this purpose. 